\title{
DISTINCIÓN ENTRE LA NATURALEZA COMÚN Y LA HECEIDAD EN DUNS SCOTO'
}

\author{
Antonio Pérez-Estévez \\ Maracaibo. Venezuela
}

\section{RESUMEN}

En este artículo se trata sobre el concepto de heceidad de Duns Scoto. El tema es analizado en las obras Lectura Oxoniensis, Ordinatio and Reportata Parisiensia.

\begin{abstract}
In this article Duns Scotus' concept of 'heceidad' is looked at, a subject open to discussion. The subject is focussed on his Lectura Oxoniensis, Ordinatio and Reportata Parisiensia.
\end{abstract}

Después de muchos textos no es fácil tener una idea precisa de lo que Duns Scoto entiende por heceidad. Dice que es una entidad formal en el género de sustancia y que pertenece a la coordinación predicamental de la sustancia primera; dice también que no es una forma añadida a la naturaleza sino la última realidad de la forma, y por lo tanto no constituye con la naturaleza el ser quiditativo del individuo. Dice que es acto o cuasi-acto de la naturaleza o entidad quaditativa y dice también que es la última realidad del ente. Es la última perfección del individuo. Posiblemente para aclarar esta aparente confusión habría que aclarar la distinción entre coordinación predicamental de la sustancia primera o individuo y quididad de la misma. Con la coordinación predicamental de un individuo expresamos todos los predicados formales de un individuo desde el género último hasta la heceidad del mismo; sería su esencia singular. Con la quididad expresamos la definición o esencia específica de un individuo. Los individuos de una misma especie tienen la misma quididad, pero distinta coordinación predicamental. La heceidad, sin ser una forma sustancial, es una cuasi forma sustancial que perfecciona la quididad y la individualiza; es la

1 Las citas de la Ordinatio y de la Lectura se hacen de acuerdo al texto crítico de la edición Vaticana: Ioannis Duns Scotus, Opera Omnia, Civitas Vaticana, 1950-1982. Reportata Parisiensia según el texto de la edición de Wadding, reimpresa en París en 1891-1895. (La traducción es mía) 
última forma de la esencia individual. A este cuadro borroso vendría a añadirse la calificación de propiedad del individuo que la Reportata atribuye a la heceidad, lo cual vendría a dificultar la comprensión de la heceidad como formando parte de la esencia individual. ${ }^{2}$

Lo que está claro es que la heceidad, sin pertenecer a la quididad o naturaleza del individuo, mantiene con ella una relación como de lo determinante a lo determinado, como de acto a potencia o como de lo más perfecto a lo menos perfecto. Ella es la causa de la unidad numeral del individuo, que es para Duns Scoto la unidad máxima y perfectísima. El individuo es el ser más perfecto — posee una perfección añadida a la naturaleza- y posee una unidad real máxima y más perfecta --la unidad de la naturaleza es real pero menor que la del individuo.

El individuo es un compuesto de naturaleza y de la heceidad y posee la unidad que producen dos entidades, una de ellas determinable, potencial y contraíble por la otra, que la determina, la actualiza y la contrae. Para explicitar este tipo de unidad que se da entre la naturaleza y la heceidad, Duns Scoto dice que pueden darse tres clases de unidades:

1. La unidad producida por la composición de cosas diversas, v.g., la composición de la materia y la forma.

2. La unidad de aquellos que se distinguen según sus razones formales, pero uno según toda su razón formal incluye al otro por identidad, aunque no formalmente y, por tanto, no es determinable y contraíble, v.g., la esencia de Dios incluye por identidad la relación de paternidad y los atributos divinos, aunque no formalmente, y la esencia no es determinable y contraíble, ni se da composición alguna entre la esencia divina y las relaciones o entre la esencia divina y los atributos.

3. La unidad que se produce en una misma cosa, que tiene diversas perfecciones o entidades formales, de tal manera que una realidad formal no incluya la otra ni formalmente es la otra, y, por tanto, la una es determinable y perfectible por la otra, tal como sucede en una cosa simple que tiene género y diferencia, v.g., en la blancura se da una unidad producida por la composición de dos formalidades distintas, a saber la del género color y la de la diferencia específica blancura.

De esta tercera clase es la composición que se da entre la naturaleza y la heceidad. ${ }^{3}$

2 Report. Parisiensia, II, d. 12, q. 8, p. 37: «nunc autem proprietas individui est minus nota nobis quam forma specifica, quia forma specifica est principium formale operandi, et non illa proprietas individui».

3 Lect. II, 3, I, 5-6, p. 285, n..$^{\circ}$ 178: «triplex est unitas: una, quae est secundum compositionem factam ex diversis rebus (sicut est compositum ex materia et forma); alia est unitas aliquorum quae distinguuntur secundum rationes suas formales, ita tamen quod unum secundum totam suam formalem rationem includat aliud per identitatem, licet non formaliter (sicut essentia et paternitas se habent in divinis et attributa [...] tamen essentia secundum suam rationem formalem includit per identitatem relationem et attributa, et ideo non est potentialis nec contrahibilis per illa; propter quod nullam compositionem faciunt); est autem unitas et distinctio media, in eadem re, quae habet diversas perfectiones sive diversas entitates formales, ita tamen quod una realitas formalis non includit aliam nec formaliter ex se est alia, et ideo est determinabilis et perfectibilis per illam, et talis est compositio in re simplici habente genus et differentiam (ut in Albedine) [...] et talis est compositio individui ex natura specifica et differentia individuali, per quam contrahitur et determinatur». 
En la Ordinatio explicita que en «todo lo común y determinable pueden aún distinguirse (en la medida que sea una cosa) múltiples realidades formalmente distintas, de las cuales ésta no es aquella: la entidad singular formalmente es ésta y la entidad de la naturaleza formalmente es aquélla. Estas dos realidades no pueden ser una cosa y una cosa, como sí pueden ser la realidad del género y la realidad de la diferencia (de las que resulta la realidad específica), pero siempre en la misma cosa (ya en la parte ya en el todo) son realidades de la misma cosa, formalmente distintas. ${ }^{4}$

Añadamos un texto de Reportata Parisiensia en el que se afirma que «no es necesario que la cosa, de la que se recibe la diferencia específica, sea distinta realmente de aquello de donde proviene el género, sin embargo no es lo mismo formalmente. Esta propiedad del individuo jamás es una cosa distinta de la forma específica, sin embargo, no es lo mismo formalmente, aunque algo pueda contener unitivamente a ambas». ${ }^{5}$

De estos textos se sacan conclusiones importantes:

1. Existe una triple unidad: a) la que se produce a partir de la composición de cosas diversas, como la composición de la materia y la forma; b) la unidad de aquellos que se distinguen por sus razones formales, de manera que una razón formal incluya a la otra por identidad, aunque no formalmente, $y$, por tanto, ni es determinable ni contraíble por la otra como sucede con la esencia y la paternidad divinas; c) la unidad y distinción media de realidades formales distintas, en una misma cosa, de manera que una no incluya la otra y, por tanto, sea determinable y perfectible por ella.

2. La distinción media c) de distintas realidades formales, la una determinable y perfectible por la otra, se da entre el género y la diferencia en una cosa simple. También se da entre la naturaleza específica y la diferencia individual o heceidad. En la Ordinatio se dice expresamente que en todo lo común y determinable, en la medida que es una cosa, pueden distinguirse formalidades distintas. En concreto la entidad de la singularidad y la entidad de la naturaleza son entidades formalmente distintas.

3. En Reportata se dice también que esta propiedad del individuo o heceidad no es una cosa distinta de la forma específica sin que sean lo mismo formalmente, es decir, que son distintas formalmente. Añade «aunque algo pueda contener unidos a ambos»; es decir, que el individuo es el continente de la naturaleza común y la heceidad, las cuales son el contenido.

4 Ordin. II, 3, I, 5-6, p. 484, n. ${ }^{\circ}$ 188: «[...] quodcumque commune, et tamen determinabile, adhuc potest distingui (quantumcumque sit una res) in plures realitates formaliter distinctas, quarum haec formaliter non est illa: et haec est formaliter entitas singularitatis, et illa est entitas naturae formaliter. Nec possunt istae duae realitates esse res et res, sicut possunt esse realitas unde accipitur genus et realitas unde accipitur differentia (ex quibus realitas specifica accipitur), sed semper in eodem (sive in parte sive in toto) sunt realitates eiusdem rei, formaliter distinctae».

5 Report. Paris., II, 12, 8, p. 37: «non necesse est rem, a qua accipitur differentia specifica, esse aliud re ab illo a quo est genus acceptum, semper tamen est non idem formaliter; sed ista proprietas individui nunquam est res alia a forma specifica, tamen semper est non idem formaliter, licet aliquid possit continere unitive utrumque». 
La unidad entre la naturaleza y la heceidad es el resultado de la composición de dos realidades formalmente distintas, en la que una es determinable y perfectible por la otra y es semejante, dice la Lectura y Reportata, a la existente entre las dos formalidades que componen una especie. En la Ordinatio señala una diferencia con el género y la diferencia específica, para sostener que la unión entre la naturaleza y la heceidad es más íntima y profunda, y será siempre, dentro de una misma cosa, eiusdem rei, la unidad de dos realidades formalmente distintas. Sin ser cosas distintas, la naturaleza común y la heceidad son formalmente distintas, aunque ambas unidas puedan estar contenidas en algo, comenta Reportata. De hecho, la naturaleza unida a la heceidad se encuentran contenidas en el individuo.

De la lectura detenida de estos textos, que acabamos de analizar, resulta difícil entender la conclusión a la que llega Woosuk Park de que «es claro que la distinción formal se da entre la heceidad y la naturaleza común individualizada en el singular». ${ }^{6}$ Es decir, para Park la distinción formal se da entre la heceidad y la naturaleza específica individualizada, o lo que es lo mismo entre la heceidad y la esencia singular, o entre la heceidad y la sustancia material individual, o entre el individuo y la heceidad, o entre el todo individual y una parte cuasiformal de ese todo.

La opinión de Park se basa en dos premisas: 1) lo que hay o existe es sólo individual; 2) la naturaleza común en sí misma no existe. ${ }^{7}$ La 2) parece ser consecuencia de la 1).

Mi opinión personal es que ambas premisas son imprecisas e inexactas, por decir lo menos, y por tanto la conclusión de que la distinción formal se da entre la naturaleza específica individualizada y la heceidad no corresponde al pensamiento de Duns Scoto.

Veamos la primera premisa: Lo que hay o existe es sólo individual. En términos scotistas habría que distinguir esta proposición:

a) acepto que lo que hay o existe con unidad numeral es sólo individual; b) niego que todo lo que hay o existe es sólo individual, ya que existen realidades, dentro de los individuos, que poseen una unidad real menor que la numeral o individual.

6 Park, Woosuk, Haecceitas and the bare Particular: A Study of Duns Scotus' Theory of Individuation, disertación multigrafiada, U.M.I., 1983, p. 175: «it is clear by now that when we talk about the formal distinction between the common nature and haecceitas, we mean the distinction between haecceitas and the common nature individuated in a material substance». También: Park, Woosuk, «Common Nature and Haecceitas», Franziskanische Studien, 71, 1989, pp.189-192. La conclusión del artículo está expresada de la siguiente manera: «it should be clear by now that when Scotus speaks about the formal distintion between the common nature and the haecceitas, he means the distinction between haecceitas and the common nature individuated in the singular».

Son dignos de destacar los matices distintos de los dos textos casi iguales: en el primero de 1983 esta opinión parece ser una interpretación de W. Park («we talk [...] we mean»), mientras que en 1988 se dice tajantemente que Duns Scoto claramente habla y significa, («scotus speaks [...] he means»).

7 En el artículo citado dice textualmente: "What there is or exists is only the individual. We can distinguish the quidditative element and the individuating element only by referring to the human mind [p. 191] [...]. Though the common nature of itself does not exist, it is independently of haecceitas» [pp. 191-92]. 
Numerosos textos de la Lectura II, 3, I, 1 afirman que existen realidades en los objetos extramentales con unidades reales menores que la numeral o individual y, por tanto, que la naturaleza común existe en el mundo de los objetos extramentales. Toda la argumentación en contra de la tesis que sostiene que la naturaleza es por sí misma singular y que viene a coincidir con lo expresado por Park en 1), se basa justamente en defender que, además de los seres con unidad real numeral, existen otras entidades en los objetos fuera de la mente con unidad real menor que la individual. La naturaleza común posee una entidad con una unidad real menor que la individual y existe en las sustancias materiales, manteniendo dicha unidad propia. Recordemos el texto de la pág. $231, \mathrm{n}^{\circ}{ }^{\circ}$, en el que dice textualmente:

[aquello] cuya unidad propia es menor que la unidad numeral, esa unidad no es numeral; ahora bien, la unidad de la naturaleza de la piedra de suyo es menor que la unidad numeral; luego la unidad de la naturaleza de la piedra, que por sí misma tiene, no es la unidad numeral. ${ }^{8}$

Obviamente, aquí se habla de la naturaleza común en sí misma, a la que se le atribuye una unidad real que no es numeral. Cuando va a demostrar la menor dice en la pág. 232: «ahora bien, la menor también es verdadera, a saber que "la unidad de la naturaleza en esta piedra es menor que la unidad numeral de esta piedra", porque si esta unidad que es de la naturaleza, no fuese menor que la unidad numeral, no existiría ninguna "unidad real" menor que la unidad numeral; el consiguiente es falso, luego también el antecedente». ${ }^{9}$ Es decir, en «esta piedra» que tiene unidad numeral existe la naturaleza «piedra» la cual tiene por sí misma una unidad numeral menor que la numeral; o dicho de otra forma, en toda sustancia material individual existe la naturaleza común, la cual mantiene en esa sustancia individual su propia unidad real menor que la numeral. Por tanto, está claro que existen extramentalmente en los individuos realidades, como la naturaleza en sí misma, con una unidad real menor que la individual. Luego, no todo lo que existe extramentalmente es sólo lo individual o singular.

Para insistir reproduzco, entre otros muchos, un texto que me parece suficientemente claro de la Ordinatio: «Así también en una cosa externa, en donde la naturaleza existe acompañada de la singularidad, no es esa naturaleza de suyo determinada a la singularidad, sino que es naturalmente anterior a la singularidad en razón de que ésta la contrae a esa singularidad, y en cuanto que es naturalmente anterior a aquello que la contrae, no le repugna ser sin aquello que la contrae. Y así como el objeto en el entendimiento según aquella su primicia y su universalidad tuvo un verdadero ser inteligible, así también en una

8 Lect. II, 3, I, 1, p. 231, n. ${ }^{\circ}$ 9: «cuius unitas propria est minor unitate numerali, illa non est unitas numeralis; sed unitas naturae lapidis de se est minor unitate numerali; ergo unitas naturae lapidis quam habet de se, non est unitas numeralis».

9 Lect. II, 3, 1, p. 232, 11: «sed minor etiam vera est quod "unitas naturae in hoc lapide est minor unitate numerali huius lapidis", quia si ista unitas quae est naturae, non esset minor unitate numerali, nulla esset "unitas realis" minor unitate numerali; consequens falsum est, ergo et antecedens». 
cosa natural según aquella entidad posee un verdadero ser real fuera del alma, y según esa entidad tiene una unidad proporcional, la cual es indiferente a la singularidad, de manera que no le repugna a esa unidad por sí misma que se una con una unidad cualquiera de la singularidad [lo entiendo de esta manera: "que la naturaleza posee una unidad real menor que la numeral"]. ${ }^{10}$

Descompongamos las afirmaciones de este texto:

1. La naturaleza existe en una cosa externa acompañada de la singularidad.

2. Esa naturaleza existente no es de suyo determinada a esa singularidad, debido a que naturalmente es anterior a esa singularidad que la contrae.

3. A esa naturaleza, como anterior, no le repugna ser sin esa singularidad.

4. La naturaleza, en una cosa natural, posee extramentalmente un verdadero ser con una unidad proporcional, indiferente a esta o a otra singularidad.

5. Esa naturaleza existente en una cosa natural posee una unidad real menor que la numeral.

En la pág. 237, n. $^{\circ} 32$, dice que «la piedra en sí misma es algo anterior al entendimiento, y no bajo la razón de universal o de singular, y cuando primero entiende piedra no la entiende como segunda intención, es decir, como universal, sino que entiende la naturaleza de piedra en sí misma, y no como universal ni como singular; de igual manera, en la existencia fuera del alma ni es primero una en número ni muchas en número, sino que tiene una unidad propia que es menor a la unidad que le conviene a aquel singular; y ése es el ser quiditativo de la piedra y en general de la cosa [...]»." Resumamos este texto:

1. La piedra en sí misma, secundum se (como naturaleza), sin razón de universal o de singular es algo anterior al entendimiento.

2. (El entendimiento), cuando entiende la piedra en primer lugar, no la entiende como segunda intención, ni la universalidad es parte de lo entendido. No entiende la naturaleza de la piedra en sí misma, secundum se, ni como universal ni como singular o particular.

10 Ordin. II. 3, I, 1, p. 404, n. ${ }^{0} 34:$ «[...] ita etiam in re extra, ubi natura est cum singularitate, non est illa natura de se determinata ad singularitatem, et in quantum est prior naturaliter illo contrahente, non repugnat sibi esse sine illo contrahente. Et sicut objectum in intellectu secundum illam primitatem eius et universalitatem habuit vere esse intelligibile, ita etiam in re natura secundum illam entitatem habet verum esse reale extra animam, et secundum illam entitatem habet unitatem sibi proportionalem, quae est indifferens at singularitatem, ita quod non repugnat illi unitati de se quod cum quacumque unitate singularitatem, ita quod non repugnat illi unitati de se quod cum quacumque unitate singularitatis ponatur (hoc igitur modo intelligo "naturam habere unitatem realem, minorem unitate numerali":)».

11 Lect. II, 3, I, 1, p. 237, n. ${ }^{\circ} 32$ : «Unde, sicut lapis prius est aliquid intellectui secundum se, et non sub ratione universalis nec sub ratione singularis, nec intelligit secundum intentionem quando primo intelligit lapidem, nec universalitas est pars intellecti, sed intelligit naturam lapidis secundum se, nec ut universalis nec ut particularis singularis, sic in exsistentia extra animam nec est primo una numero nec multa numero, sed habet unitatem propriam sibi, quae minor est unitate quae convenit illi singulari; et illud est "esse" quiditativum lapidis et rei universaliter». 
3. (La naturaleza en sí misma) en la existencia fuera del alma ni es una numéricamente ni múltiple numéricamente, sino que tiene una unidad propia que es menor que la unidad de aquel singular.

4. Aquello (la naturaleza en sí misma) es «el ser» quiditativo de la piedra y, en general, de la cosa.

La naturaleza en sí misma o común, que es el ser quiditativo de cualquier sustancia material o cosa, existe en la realidad extramental antes de que la entendamos o conozcamos con el entendimiento. Y existe sin ser ni universal ni singular. ${ }^{12}$ Cuando primero conocemos la naturaleza en sí misma, no la conocemos como segunda intención, es decir, como universal. La naturaleza en sí misma posee en la realidad extramental una unidad real menor que la numeral. De acuerdo a estos textos es difícil entender las afirmaciones 1) y 2) de Park de que, para Duns Scoto, lo que existe es sólo individual y de que la naturaleza en sí misma no existe extramentalmente. De aquí que me parezca falsa, a partir de estos mismo textos, la afirmación de Park de que la distinción entre el elemento quiditativo o naturaleza común y el elemento individuante diga referencia sólo a la mente humana. ${ }^{13}$ En el último texto está suficientemente claro que el elemento quiditativo es la naturaleza en sí misma y el elemento individuante la heceidad, y que ambos elementos se dan en la realidad extramental como formalidades distintas.

Si las dos premisas utilizadas por Park nos parecen, a partir de estos textos, falsas, la conclusión nos parece también falsa. Es decir la conclusión de que la distinción formal de Scoto se da entre la «la naturaleza común individualizada y la heceidad». Veamos.

En el texto de la Lectura (II, 3, I, 5-6, p. 285, 178) se dice expresamente que esta distinción media o formal «se da en una misma cosa que tiene diversas perfecciones o diversas entidades formales, de manera que una realidad formal no incluye a la otra ni es de suyo formalmente la otra, y, por tanto, es determinable y perfectible por ella». Termina diciendo unas líneas después: «el individuo se compone de naturaleza específica y diferencia individual, por la que [aquélla] se contrae y se individualiza». La distinción formal se da en una misma cosa entre dos entidades formales, de las cuales una es determinable y perfectible y la otra determina y perfecciona a la primera.

12 Esta opinión, que por lo demás ha sido común entre los intérpretes de Duns Scoto, la refleja Alberto Squittieri, cuando, hablando de la naturaleza común, dice: «e per l'altro nelle sostanze materiali individuali in cui è numericamente ripprodota, la natura non è per se stessa singolare o determinata alla singolarità, ma possiede unasua propria unità essenziale precedente e indifferente alla causa che la contrae all'individualità». («La Definizione del Principio di Individuazione in Duns Scoto: Analisi delle Difficoltà», Studia Patavina, 34 Padova, 1987, p. 9).

También T.M. Radovsky, «The Doctrine of Individuation in Duns Scotus», Franziskanische Studien, 62, 1980, p. 68: «In any individual the common nature has a two-fold unity: first, a unity that belongs to it not of itself but on account of the individual in which it is found; and second, the minor unity that belongs to it of itself».

13 Park, W., «Common Nature», p. 191: «We can discern the quidditative element and the individuating element only by referring to the human mind». 
La naturaleza común en sí misma, ha dicho repetidas veces, es determinable, en potencia y perfectible por la heceidad. Y ésta determina, actualiza y perfecciona a la naturaleza común en sí misma. Por tanto, es entre la naturaleza común en sí misma y la heciedad que se da la distinción formal. «La naturaleza común individualizada» no es determinable, en potencia y perfectible con respecto a la heceidad, sino que ya se encuentra determinada, actualizada y perfeccionada por ella. Por tanto, no puede darse la distinción formal entre la «naturaleza común individualizada» y la heceidad. Nos parece obvio que Duns Scoto habla en los textos citados de la naturaleza común — no individualizada—, la cual está con respecto a la heceidad en potencia para ser determinada y perfeccionada. Esta opinión nuestra implica la aceptación de que en el individuo existen dos naturalezas, la naturaleza común y la naturaleza individualizada, tal como expresamente lo afirma Duns Scoto cuando dice: «en el individuo existe la naturaleza y esta naturaleza, y el individuo añade a la naturaleza absoluta aquella realidad formal por la que tiene unidad propia y singular». ${ }^{1+}$

La naturaleza común exiște en el individuo y lo hace con la unidad que le es propia, y que, como sabemos, es una unidad real menor que la singular; y la heceidad es una forma o cuasi-forma, añadida a la naturaleza común, que la perfecciona, la contrae y la actualiza. También existe en el individuo, constituyéndolo, la naturaleza común individualizada o «esta naturaleza».

El mundo creado de Duns Scoto es un conjunto de sustancias individuales, en las que existen —además de cosas (res) distintas, como la materia y la forma— entidades formales, como la naturaleza común, que conserva en el individuo su unidad propia real menor que la individual. La unidad máxima y más perfecta del singular creado scotista es el resultado de la composición de múltiples unidades reales incompletas e imperfectas, que se encuentran encerradas en esa unidad perfecta del individuo; la de la naturaleza es menor que la singular.

Si esto es así, en ese caso tanto el artículo citado de Woosuk Park como su tesis doctoral, Haecceitas and the bare Particular, ${ }^{15}$ contienen numerosas imprecisiones. Nos parece falso, como ya hemos demostrado, afirmar que, para Duns Scoto, la naturaleza común en sí misma no existe, tal como Park afirma: «though the common nature in itself does not exist» (pp. 191-92). Nos parecen igualmente falsas las afirmaciones hechas en la p. 192, por las que se pretende que la naturaleza común no cumple los requisitos exigidos por la definición formal tomada de Jordan, a saber:

1. «La naturaleza común en sí misma y la heceidad no se dan en una misma cosa», pues nos parece haber demostrado en los textos anteriores que «la naturaleza común en sí misma existe en las cosas individuales materiales, acompañada de la heceidad». ${ }^{16}$

14 Lect. II, 3, I, 5-6, p. 286, n. ${ }^{\circ}$ 179: «ita in individuo est natura et haec natura, et individuum addit super naturam absolute illam realitatem formalem per quam habet unitatem propriam et singularitatem [...]».

15 Park, Woosuk, Haecceitas and the bare Particular: A study' of duns Scotus' Theory of Individuation, cit. en nota 6.

16 Park, W., «Common Nature», p. 192: «The common nature in itself and haecceitas are not fount in a single thing». 
2. «Es imposible decir que la naturaleza común en sí misma y la heceidad son realmente idénticas». ${ }^{17}$ No solo es posible decirlo, sino que, de acuerdo a los textos expuestos, Duns Scoto afirma que «la naturaleza común en sí misma y la heceidad son realmente idénticas y formalmente distintas». 18

3. «Si la naturaleza común en sí misma y la heceidad son distintas, tienen que ser realmente distintas». Es decir, la diferencia entre la naturaleza común y la heceidad sería, en opinión de Park, la existente entre res y res, entre cosas distintas. ${ }^{19}$ Creemos haber demostrado hermenéuticamente que la naturaleza y la heceidad se distinguen formalmente.

Esta opinión 3 no es nueva. Ya en el siglo XIV algunos autores como Guillermo de Alnwick se la atribuían a Duns Scoto. Guillermo de Ockham le dice «opinión impuesta por algunos al Doctor Sutil» «opinio, quae imponitur doctori subtili a quibusdam», y la resume de la siguiente manera: «Y hay la opinión de que el universal es una verdadera cosa fuera del alma, distinta realmente de una diferencia que la contrae, sin embargo multiplicada en varios por medio de esa diferencia que la contrae». ${ }^{20}$ Este universal es incompleto y potencial.

La genuina doctrina de Duns Scoto, para Guillermo de Ockham, es la que dice «que en la cosa fuera del alma existe la naturaleza idéntica realmente con la diferencia que la contrae a determinado individuo, pero formalmente distinta; [naturaleza] que de suyo no es ni universal ni particular, sino que es universal incompleto en la cosa y completo según el ser en el entendimiento» y que coincide fundamentalmente con la lectura que nosotros hemos hecho de los textos scotistas. ${ }^{21}$

Finalmente, echando una ojeada en perspectiva, nos parece que el origen de la confusión en Woosuk Park es suponer que «la naturaleza en sí misma equivale a naturaleza independiente de la heceidad» en el sentido de tener un ser autónomo capaz de existir separada de la heceidad..$^{22}$ Efectivamente, esa natualeza, así entendida, ni existe en la realidad extramental, ni creemos que exista en los textos de Duns Scoto.

Antonio Pérez-Estévez

Apartado 10114

Maracaibo (Venezuela)

17 Ibid. p. 192: «It is impossible to say that the common nature in itself and haecceitas are really identical».

18 Report. Paris., II, 12, 8, p. 40: «Nunc autem proprietas indivisibilis, quanquam sit posterior natura quidditate, nunquam tamen est res alia, sed est idem identitate cum forma specifica, quamvis alia formalitas».

19 Park, W., «Common Nature», p. 192: «Thus, if they are distinct, they would have to be really distinct».

20 Guillelmi de Ockham. Scriptum in Librum primum Sententiarum Ordinatio, edición de Stephanus Brown, O.F.M., St. Bonaventure, New York, 1970, liber I, dist. 2, q. V, p. 154: «Et est opinio quod universale est res vera extra animam, distincta realiter ab una differentia contrahente, realiter tamen multiplicata et variata per talem differentiam contrahentem».

21 Ibid., I, 2. VI, p. 161: «dicitur quod in re extra animam est natura eadem realiter cum differentia contrahente ad determinatum individuum, disincta tamen formaliter, quae de se nec est universalis nec particularis, sed incomplete est universalis in re complete secundum esse in intellectu. Et quia ista opinio est, ut credo, opinio Subtilis Doctoris [...]».

22 Tal es el sentido que parece tener el tex to de W. Park: «the common nature absolutely considered does not exist in this spatio-temporal world, it is independently of haecceitas» (Haecceitas, p. 174). 